\title{
Staining of the conjunctiva and conjunctival tear
}

\section{film}

\author{
Joseph A Eliason, David M Maurice
}

\begin{abstract}
Both the distribution of tear fluid over the conjunctiva and any injury to the conjunctival epithelium can be made visible by instilling a fluorescent solution into the eye and observing with an appropriate combination of excitation and barrier filters. Sulphorhodamine B, which has an orange fluorescence that can be separated from the green natural fluorescence of the ocular tissues, gives a greater contrast than fluorescein. The tear film is seen to cover the surface of the conjunctiva and to be concentrated in its folds. Small circular areas of thin tear film appear transiently in the neighbourhood of the limbus after a blink. Occasional cells stain on the normal conjunctiva, particularly in the interpalpebral area. The density of the staining increases in dry eye conditions. Conjunctival trauma is sensitively revealed by the method, and its healing can be followed. Hard contact lenses are seen to traumatise continually the inferior limbal conjunctiva in symptomless wearers.
\end{abstract}

The instillation of a drop of fluorescein is a common method of detecting injury to the corneal epithelium. Before damaged cells are revealed by the stain, the precorneal tear film is coloured by the dye, and it is possible to examine it for normal variations in uniformity or for the appearance of dark patches when the layer breaks up. The conjunctival cells and the preconjunctival tear film may be of equal physiological and pathological interest to that of the corneal epithelium, but no equivalent method of staining them has been available. Rose Bengal is employed as a stain for conjunctival damage, but it provides poor contrast as usually applied, and is uncomfortable when instilled in the eye.

There are three reasons why staining with fluorescein has not been found valuable over the conjunctival surface:

(1) The use of an inefficient optical filter system. Generally, a cobalt filter has been placed in front of the slit-lamp light and the eye has been observed under the microscope without the intervention of a barrier filter. This is adequate for the observation over the dark background of the cornea, but the scattering of the incident blue light by the sclera hides the weaker fluorescent light emitted by the dye.

(2) The background fluorescence of the tissue. Even with an efficient pair of excitation and barrier filters the blue incident light will excite a greenish fluorescence in the conjunctiva and sclera, which is seen as a general glow that reduces the contrast of the fluorescein.

(3) Penetration of fluorescein. Because of the slight lipid solubility of the dye, it can stain the conjunctiva tissue appreciably, and this further reduces the contrast between the surface and the background.

It was thought possible that problem (3) could be overcome by the use of a more hydrophilic dye such as carboxyfluorescein, but it was decided to evade problem (2) as well by using the fluorophore, sulphorhodamine $B$, which is not only very hydrophilic but can be excited by wavelengths too long to lead to visible fluorescence in the tissues. When observed with an appropriate pair of colour filters, it allowed both the tear film and devitalised conjunctival cells to be readily observed with high contrast against the tissue background.

The purpose of this report is to emphasise the advantages of an appropriate pair of colour filters when fluorescent dyes are used to examine the conjunctival surface, and to illustrate the additional benefit of using a dye with larger wavelength fluorescent characteristics than fluorescein. In addition, we note some hitherto undescribed physiological and anatomical properties of the conjunctival tear film and survey some clinical conditions in which the technique might prove to have value. (These observations have already been briefly noted in the abstracts of the First Meeting of the International Society of Dacriology, Budapest 1987, and ARVO 1988.)

\section{Materials and methods}

Sulphorhodamine B (molecular weight 559) is similar in chemical structure and properties to fluorescein but is less lipid soluble, with an octanol/water partition coefficient of 0.0033 compared with 0.61 for fluorescein. 'Its fluorescence is orange and is most readily excited by green light, the peak absorption and emission wavelengths being 556 and $572 \mathrm{~nm}$. It is usually supplied as an acid and must be dissolved in alkali, being soluble to the extent of about $20 \mathrm{~g} / \mathrm{lat} \mathrm{pH}$ 7. The toxicity of the compound appears to be very low. Doses of up to $1 \mathrm{~g} / \mathrm{kg}$ could be injected intraperitoneally in the mouse without causing death, and $50 \mu \mathrm{l}$ of the saturated solution injected into the stroma of a rabbit cornea had as little effect as an equal volume of saline.

The subjects were patients, residents, and staff of the ophthalmology clinic. Informed consent was obtained after the nature of the procedure had been fully explained. A $0.5 \%$ solution of the dye at $\mathrm{pH} 7$, made $300 \mathrm{mM}$ with sucrose, was used to stain the tear film and a small drop was applied to the tarsal conjunctiva with a wooden applicator. The solution did not cause discomfort in the eye of any volunteer or patient. It is not important, however, to adhere exactly to this dye formulation.

The eye was observed with a Haag-Streit 900 slit-lamp using a broad patch of illumination. A band-pass interference filter of $520-550 \mathrm{~nm}$, such as is used as a barrier filter for fluorescein, was inserted in the illuminating light path, and gelatin absorption filters (Kodak No 22) transmitting $100 \%$ of the light at $600 \mathrm{~nm}$ were placed in the eyepieces. This combination of filters passes less than $0.01 \%$ of light at the cross-over point of their absorption curves, around $570 \mathrm{~nm}$. Photographs were taken with a Zeiss photoflash slit-lamp on 1600 ASA colour film with the same combination of filters. Black-and-white film of similar speed can also be used. 
Figure 1 Staining of tear film with sulphorhodamine $B$ in a normal subject. ((A) Pattern over conjunctiva immediately after a blink. (B) Same area of conjunctiva a few seconds later. Note the enhancement of lines and patches with time.

Figure 2 Disappearance of conjunctival pattern with gentle pressure from a transparent plastic plate (arrow).

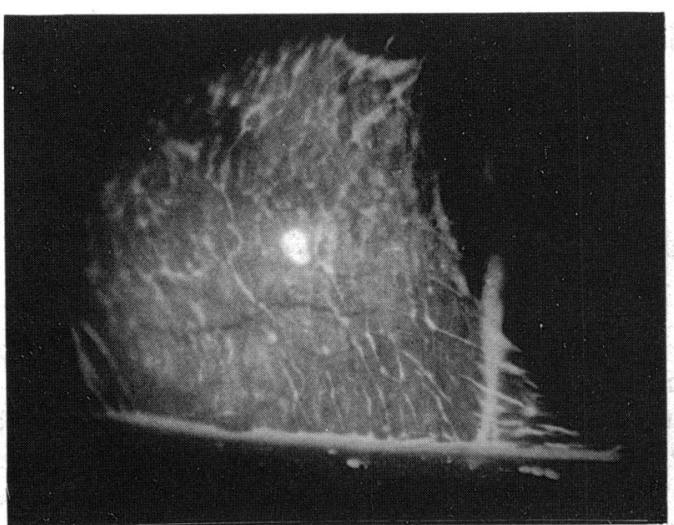

Figure 1A

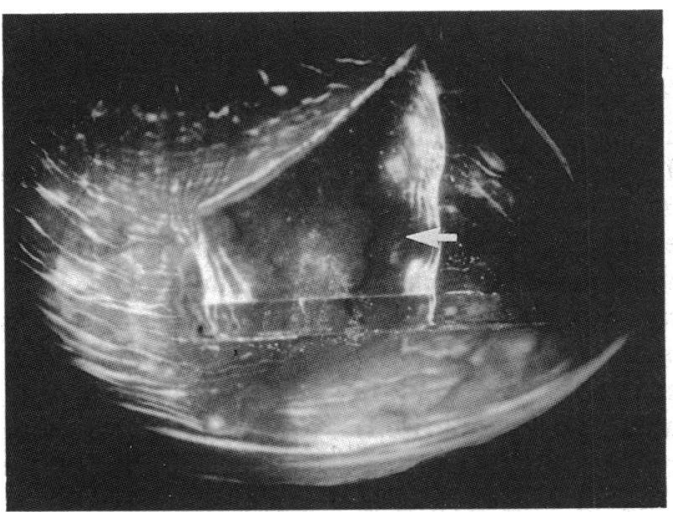

Results

NORMAL TEAR FILM

In the absence of the dye the tissues of the eye appeared black through the microscope, making focusing on the ocular surface difficult. Pingueculae gave rise to a natural fluorescence which was absent in normal sclera or cornea.

After the dye was instilled the precorneal tear film and the lacrimal strips shone as brilliantly as they do with fluorescein under blue illumination. Furthermore, the conjunctiva was seen, in the first moments after instillation, to be covered with a more or less uniform tear film. Shortly thereafter a system of lines and patches appeared which became more prominent during the first seconds immediately after opening the eye, while the fluorescence of the tear film covering the area between them became weaker (Figs 1A, B). These seem to be a result of accumulations of

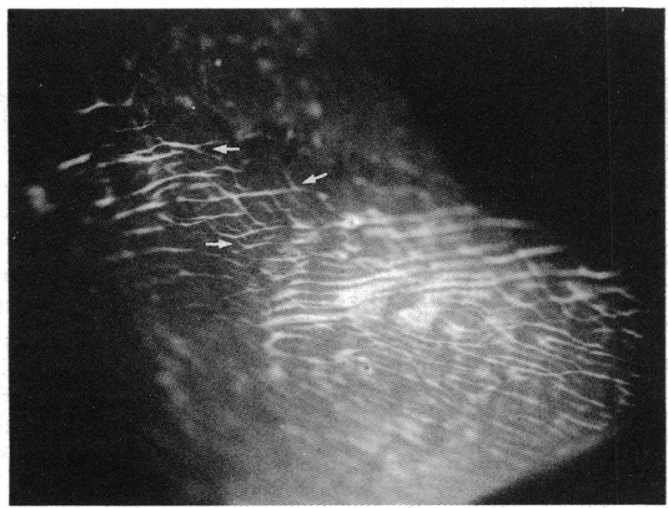

Figure $3 A$

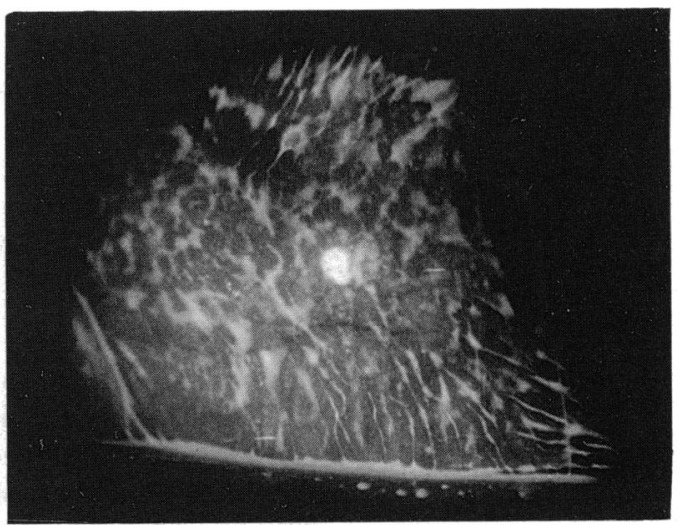

Figure $1 B$

fluid rather than tissue staining, since they disappeared after thorough flushing with saline or on pressing the conjunctiva under a clear plastic plate (Fig 2). Many features of the patterns of the lines remained constant over a long period, and they almost certainly correspond to folds in the conjunctiva (Figs $3 \mathrm{~A}, \mathrm{~B}$ ). The wider patches may represent layers of mucus or broad depressions in the conjunctival surface. The thickness of the mucus layer on the conjunctiva has been reported to vary over the surface. $^{2}$

Some transient phenomena occur after a blink. The tear film over the entire cornea and conjunctiva appeared to flow upwards immediately after the opening of the eye. This movement lasted for about 3 seconds. Often circular dark patches about $1 \mathrm{~mm}$ in diameter were observed in the tear film over the conjunctiva and cornea, most frequently near the limbus (Fig 4). They appeared immediately after a blink, flowed upward with the tear film, and vanished in a second or two by constricting to smaller diameters. The origin of these spots has not been examined, but it seems most likely that they would correspond to an area where the aqueous layer is thinner rather than where it is diluted.

\section{CONJUNCTIVAL STAINING}

If the dye was removed from the tear film by thorough flushing with saline, or if it was allowed time to be washed out in the normal tear secretions, the background that remained was either dark or showed a faint orange glow from dye that

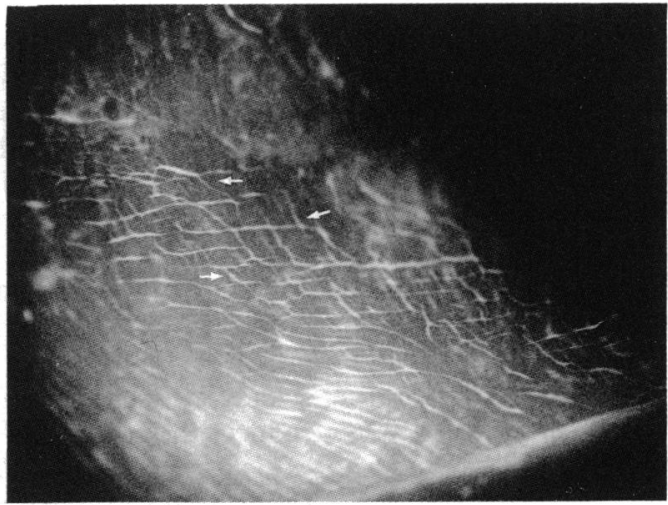

Figure 3B 


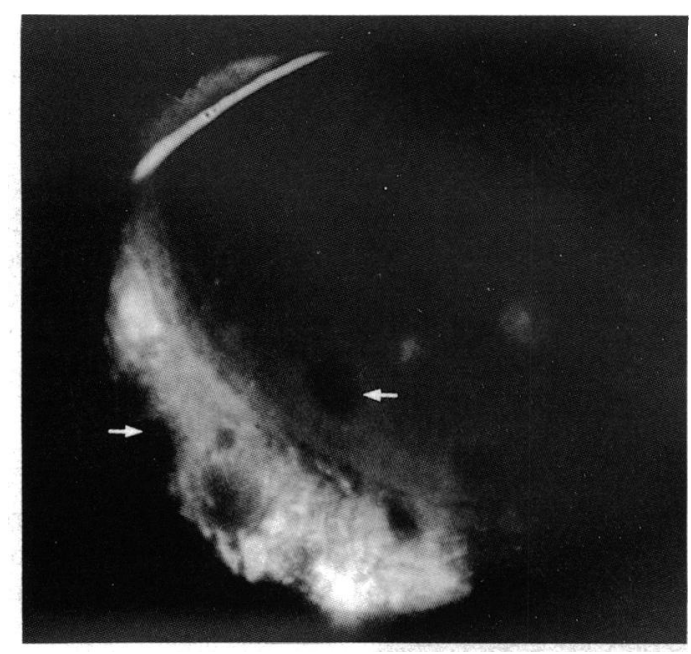

Figure 4 Dark patches (arrows) noticed here on both sides of the limbus, which were observed to move with the tear film.

Figure 5 Conjunctival staining along the limbus in the interpalpebral zone in a normal eye. Also note prominent staining of the tear meniscus along the lower lid margin.
Figure 6: Sulphorhodamine $B$ staining of the conjunctiva in a normal subject. (A) damage stained immediately after its production. (B) after its production. (B)
Restained 24 hours later, showing almost complete healing of the defect.

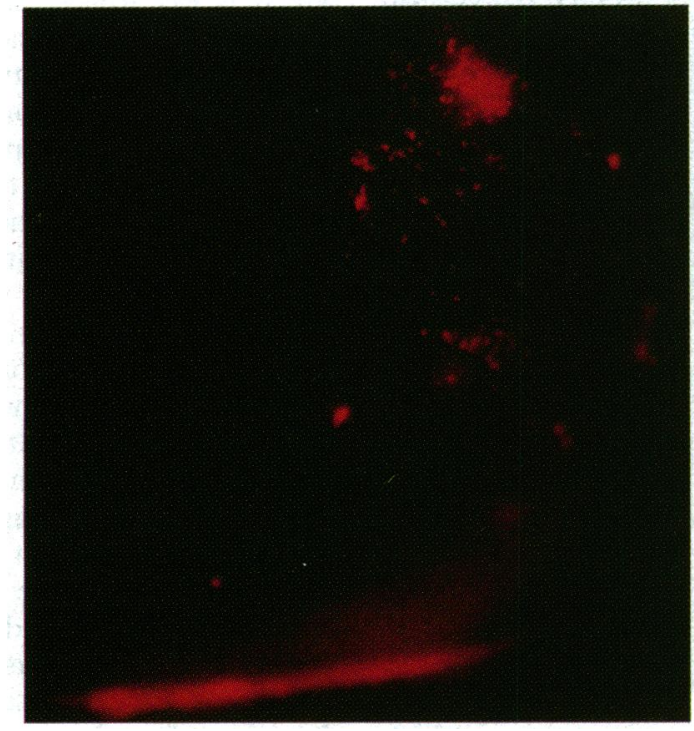

had penetrated into the tissue. In the normal eye bright staining spots were seen, occasionally on the cornea but more frequently over the conjunctiva, particularly the interpalpebral zone (Fig 5). Sometimes small patches of staining were observed. The spots retained sharp outlines and could be observed for hours, though usually they disappeared by the next day and were not

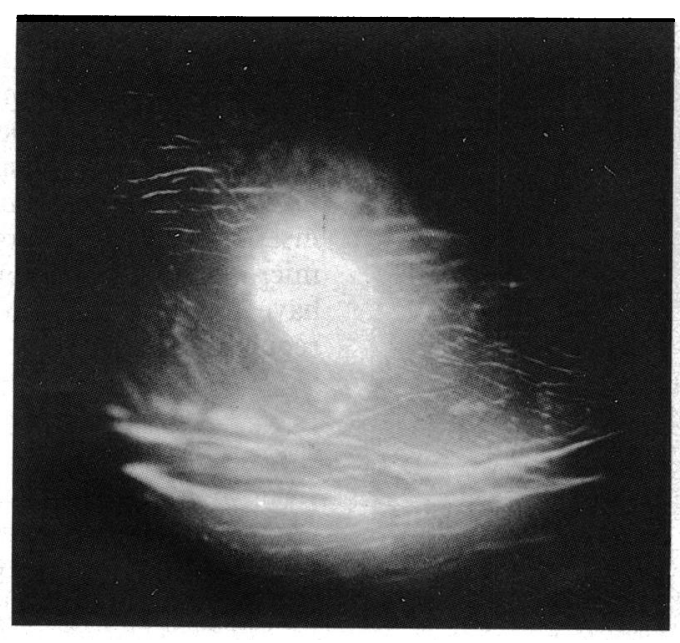

Figure $6 A$ constant on restaining the tissue. They probably correspond to individual epithelial cells that are either damaged or in a permeable condition before desquamation. Patches of staining also appeared where the tarsal or bulbar conjunctiva had been touched by the applicator. In one eye a brilliantly staining zone of conjunctival damage was created by the rotation of the wooden end of a sterile Q-tip, and its recovery healing could be followed by restaining at intervals (Fig 6A, B). In this case a portion of the initially applied stain was still present after 24 hours.

In each one of four symptomless gas permeable contact-lens wearers notable staining in the form of a confluent band superimposed on a fine line was found on the conjunctival side of the inferior limbus (Fig 7).

\section{COMPARISON WITH FLUORESCEIN AND ROSE} BENGAL

In 12 patients with Sjøgren's syndrome conjunctival staining with sulphorhodamine B was found to be more evident than that detected with fluorescein (Figs 8A, B). Corneal staining was similar with both dyes. This subjective impression that sulphorhodamine B staining was more sensitive than that with the other dyes was given a quantitative test. Observations were carried out on 14 eyes of seven normal subjects with $1 \%$ fluorescein, $0.5 \%$ rose Bengal, and $0.5 \%$ sulphorhodamine $\mathrm{B}$. The blue and green excitation filters provided with the Haag-Streit 900 slitlamp were used to observe the fluorescein and rose Bengal dyes, and the excitation and barrier filters described above were used for the sulphorhodamine. While this arrangement should provide better contrast for viewing the sulphorhodamine, the recognition of punctate staining spots over the conjunctiva with fluorescein was judged similar with or without the use of a barrier filter. Punctate staining was quantitated by the grading scheme described by van Bijsterveld $^{3}$ using four areas: cornea, and nasal, temporal and inferior conjunctiva. Each area was graded on a 0 to 3 scale: $0=$ no punctate staining; $1=$ small (that is, readily countable) number of punctate staining spots; $2=$ numerous (that is, not countable) spots; and $3=$ staining that was confluent or approaching confluence. The scores for each eye were added to reach a total of 0 to 12 .

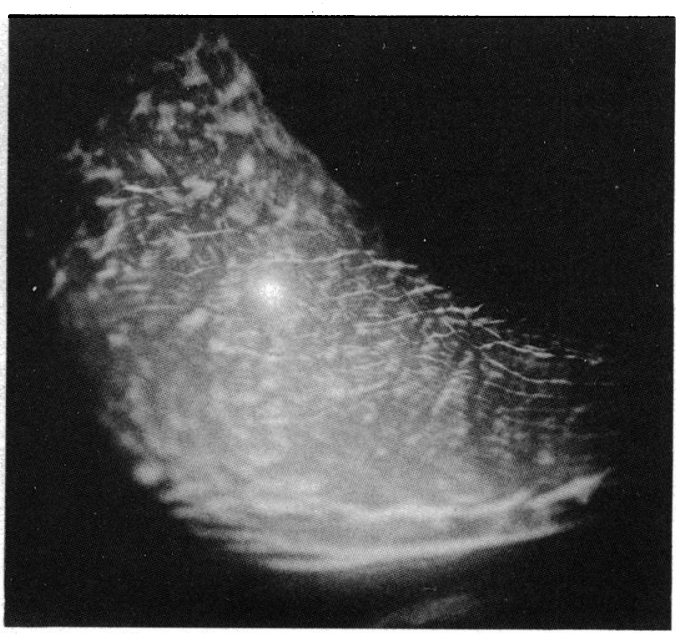

Figure $6 B$ 

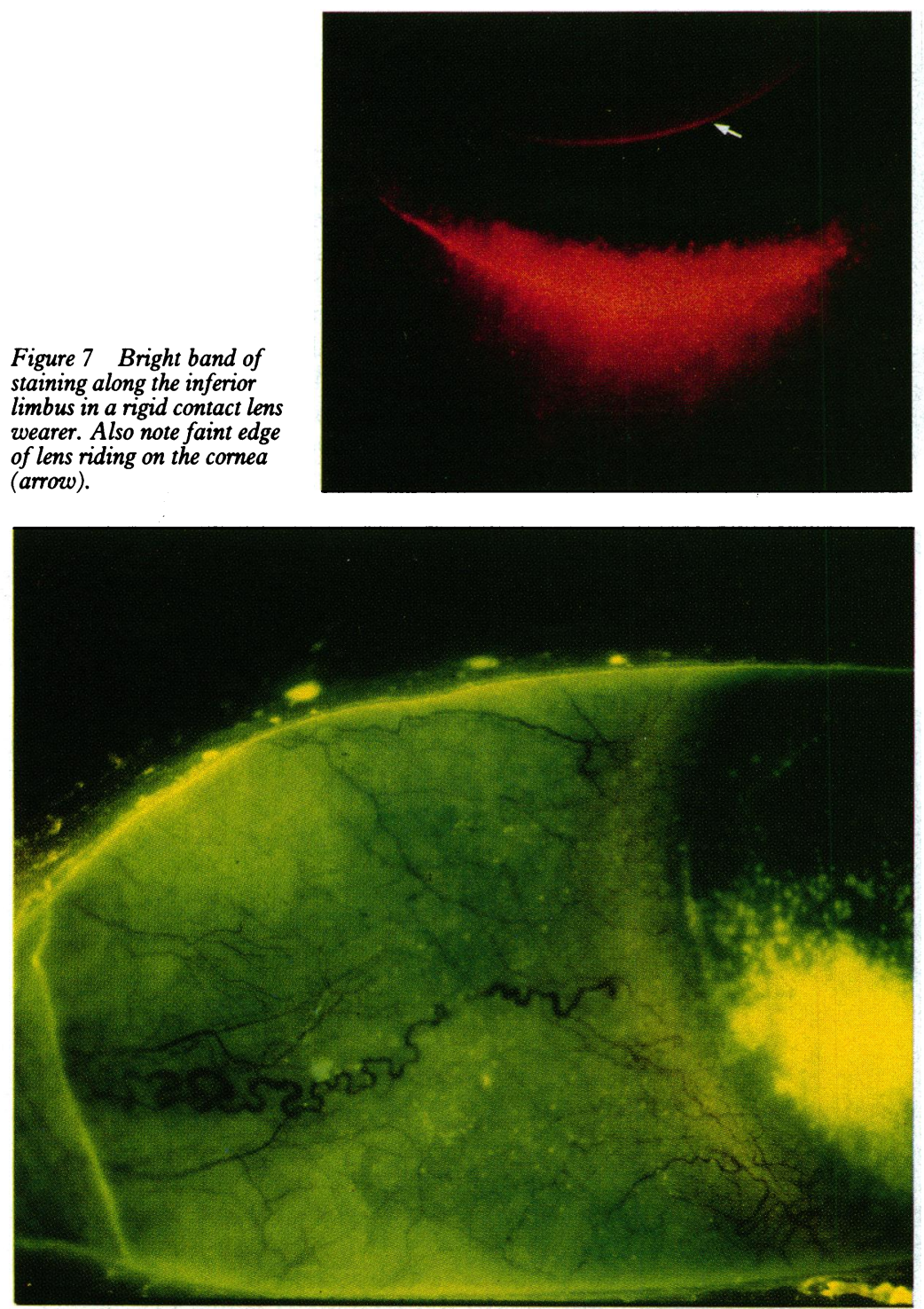

Figure $8 A$

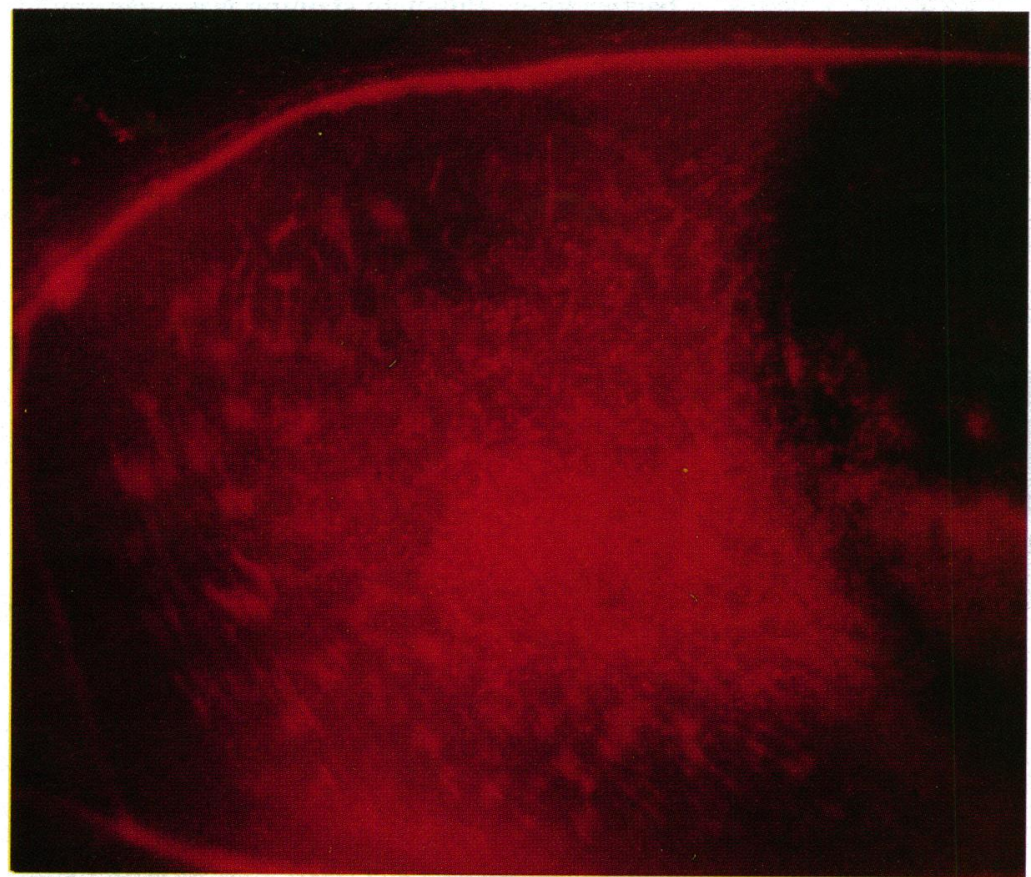

Figure $8 B$

Figure 8 (A) Conjunctival staining in a patient with primary Sjøgren's syndrome by means of fluorescein photographed with excitation and barrier filters. (B) Same location with sulphorhodamine B. Note large area of confluent staining in the interpalpebral fissure area not evident with fluorescein and absence of distracting blood vessels.
Mean scores for the dyes were : fluorescein $1 \cdot 0$, rose Bengal $1 \cdot 2$, and sulphorhodamine $2 \cdot 7$. Evaluated with the non-parametric Wilcoxon signed rank test, the difference between the fluorescein and rose Bengal scores was significant only at the $5 \%$ level, while the differences between either fluorescein or rose Bengal and sulphorhodamine were significant at the $1 \%$ level.

\section{Discussion}

Fluorescein staining of the cornea or the precorneal tear film can be readily observed under a white light or with a cobalt filter, but the dye is scarcely visible on the conjunctival surface. The use of an efficient pair of excitation and barrier filters leads to a considerable increase in contrast of the dye and allows conjunctival lesions to be identified. However, the blood vessels of the conjunctiva show up as a system of black lines which is disturbing, and background fluorescence is appreciably greater following washout of the dye, so that contrast is poorer and the detection of small lesions is difficult. The lines are not visible when sulphorhodamine $B$ replaces fluorescein, and the contrast over the conjunctiva is much improved. The colouration with rose Bengal observed in white light seemed to be the weakest of all, but it was more visible when excited with green light.

From our comparison of the three dyes we consider that sulphorhodamine $B$ is the most capable of revealing subtle surface abnormalities. Although fluorescein under our operating conditions resulted in an image easier to photograph, this is not an important consideration for visual observations. Punctate staining with sulphorhodamine B could be detected in all eyes considered normal and asymptomatic which we examined. An increase in the sensitivity of detection of ocular surface abnormalities may result in new diagnostic insights. Thus the evidence of limbal epithelial damage in asymptomatic contact lens wearers we believe to be original. It is probable that this considerable epithelial injury occurs when the edge of the lens is driven down on to the limbus on blinking. Damage to the important repopulating limbal epithelial cells may explain the poor surface healing behaviour observed in long-term lens wearers.

Further experience is required to determine whether this increase in sensitivity will lead to an improvement in diagnosis sufficient to warrant the general use of sulphorhodamine B. An important prerequisite is a method of readily inserting colour filters in the lamp and the microscope optical pathways. The system we have employed is too slow and awkward for regular use.

This work was supported by EY 04863 and Research to Preven Blindness, Inc.

1 Araie M, Maurice D. The rate of diffusion of fluorophore through the corneal epithelium and stroma. Exp Eye Res 1987; 44: 73-87.

2 Nichols BA, Chiappino ML, Dawson CR. Demonstration of the mucous layer of the tear films by electron microscopy. Invest mucous layer of the tear films by electron microscopy. Invest

3 van Bijsterveld OP. Diagnostic tests in the sicca syndrome. Arch Ophthalmol 1969; 82: 10-4. 